\title{
Finding the origin
}

Using lineage tracing techniques, Kunyoo Shin, Philip Beachy and colleagues have tracked the development of invasive bladder cancer in mice and show that basal stem cells, which express sonic hedgehog $(S h h)$, initiate this disease, but the expression of Shh is lost as the disease progresses.

The authors used N-butyl-N4-hydroxybutyl nitrosamine (BBN), which is carcinogenic specifically in the bladder, to induce mutations within the urothelium (the simple transitional epithelium that covers the bladder lumen). BBN is one of the carcinogens present in tobacco, and smoking substantially increases the risk of developing bladder cancer. Lesions that are histologically identical to human bladder carcinoma in situ (CIS) are evident by 3 to 4 months in mice exposed to BBN through their drinking water and these progress to muscle-invasive lesions by 6 months. The authors used tamoxifeninducible Cre-Lox methods to label basal Shh-expressing cells (thought to be stem cells) and their progeny with membrane-bound enhanced green fluorescent protein (EGFP) and found that marked cells were present in CIS lesions, despite an abundance of unmarked suprabasal and luminal cells at the start of exposure to BBN. Specific ablation of the Shh-expressing basal cells prevented the development of invasive bladder cancer in mice exposed to BBN and substantially reduced the incidence of CIS, indicating that these cells are essential for invasive tumour development. In addition, the integrity of the urothelium was compromised over time owing to a lack of epithelial renewal, which is consistent with Shh-expressing basal cells containing the stem cell population.

The authors also labelled the Shh basal cell population at later stages of cancer progression. Tamoxifen-induced expression of EGFP in established CIS lesions showed that Shh expression was maintained in a subset of basal cells, and serial transplantation assays in immunocompromised mice showed that these Shh-expressing cells were tumour propagating cells. Interestingly, in mice with muscleinvasive lesions, Shh expression was lost (no cells became labelled with membrane-bound EGFP on exposure to tamoxifen to induce Cre recombination, and $S h h$ mRNA was also undetectable in these lesions). These findings are in agreement with a lack of $\mathrm{SHH}$ mRNA expression in human invasive bladder cancer.

To investigate the clonality of bladder cancer, the authors used 'Rainbow mice', in which EGFP is initially expressed in all cells but the expression of one of three other fluorescent proteins can be induced in individual cells through tamoxifen to induce Cre-mediated recombination. The treatment of these mice with BBN after Cre recombination indicated that the majority of CIS lesions are clonal, with a few mice presenting with oligoclonal tumours. This pattern is maintained in invasive lesions. The authors also observed that the precancerous clonal and oligoclonal cells rapidly colonized the surrounding urothelium.

These findings indicate a model in which Shh-expressing basal stem cells are essential for the development of CIS lesions that can progress to invasive carcinoma. Why Shh expression is lost at the stage of invasive growth is not yet clear. These findings also indicate that surgery for invasive bladder cancer might not remove all of the cells with the capacity to generate invasive bladder cancer and might explain the frequent recurrence of disease in these patients and the associated high morbidity.

Nicola McCarthy

ORIGINAL RESEARCH PAPER Shin, K. et al. Cellular origin of bladder neoplasia and tissue dynamics of its progression to invasive carcinoma. Nature Cell Biol. 16, 469-478 (2014) 